\title{
Investigation of a Molecular Plasma from its Acoustic Response
}

\author{
Gilles Courret, Petri Nikkola, Mirko Croci, and Peter W. Egolf
}

\begin{abstract}
In developing a pulsed microwave lamp of $1 \mathrm{~kW}$, fitted with a static electrodeless spherical bulb of glass, we discovered a phenomenon in which the plasma forms a ball at the bulb's center, despite the effect of gravity. The light is produced by a plasma of sulfur, which is so hot that it might melt the bulb if it remained in contact with the glass, although it is fused quartz. In a preceding publication, we reported on photometric measurements showing the plasma response to the modulation of the microwaves. We have shown that the ball formation results from an acoustic resonance in a spherical mode. From a measurement of the resonance frequency, we have assessed the average value of the pressure inside the bulb. The plasma ball formation has been reproduced by another team of researchers with a similar setup, but with a spinning bulb in order to stabilize the ball. From measurements of the oscillations of the ball diameter, they have shown that the acoustic amplitude inside the bulb can reach $180 \mathrm{~dB}$ (re $20 \mu \mathrm{Pa}$ ). In our set-up, we have not measured this feature. However, improving our model of the plasma modulation, we have also been able to assess the acoustic amplitude, and we have found results in agreement, as shown in this article. In addition, our analysis suggests that a stimulated emission is involved in the observed fast time scale modulation of the light emission.
\end{abstract}

Index Terms - plasma lamp, light generation, acoustic waves, plasma sound waves.

\section{INTRODUCTION}

A MONG various plasma applications, light generation is probably the most common one. Fluorescent tubes are widely used in public spaces and households, although they contain mercury. Solid-state lighting is increasingly taking over, but there is also electrodeless plasma lighting technology without harmful substances, using microwave induction. The degree of ionization is weak and the plasma molecular [1], page 22. The emitted spectrum is almost exclusively in the visible part, Fig.A.2 [2], making the light source highly efficient. In an applied research project on the development of a such a pulsed microwave sulfur lamp prototype of $1 \mathrm{~kW}$, we have discovered a phenomenon in which the plasma forms a ball staying at the center of the bulb despite the effect of gravity, thus, protecting the fused quartz bulb from melting (cf. Fig. 1). This glass is characterized by resistance to high temperatures and offers great transparency in the optical [3] as well as in the microwave range [4].

The main mechanism of the light emission results from the sulfur recombination, which produces diatoms in an electronic excited state of radiative de-excitation ${ }^{*} \mathrm{~S}_{2} \rightarrow \mathrm{S}_{2}+\mathrm{h} \nu$

G. Courret, P. Nikkola, and M. Croci are with the University of Applied Sciences and Arts Western Switzerland, Yverdon-les-Bains, Switzerland.

P. W. Egolf is with the Institute of Theoretical Turbulence Research, Niederlenz, Switzerland.

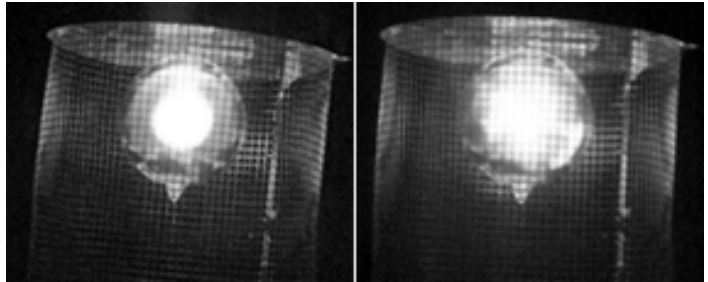

Fig. 1. Left: The plasma in spherical resonance has a spherical shape and, despite gravitation, is confined in the middle of the bulb. Right: Plasma out of resonance has an irregular shape accompanied with random ejections of plasma.

[1], page 15, and [5]. The plasma response to short pulses is measured with a fast photodiode. The signal shows beats at the spherical resonance, as reported in the previous publication [2]. The beats could result from the simultaneous excitation of two normal modes with a frequency difference of approximately $1 \%$, as shown in Fig. 2. One of the two frequencies matches precisely the repetition frequency of the microwave pulses $f_{c}$, slightly below $30 \mathrm{kHz}$. Thus, this frequency is due to a forced oscillation, whereas the other one is due to a free oscillation. We have found experimentally that this repetition frequency of about $30 \mathrm{kHz}$ of the microwave pulses is most probably to bring the plasma in the spherical resonance. If the repetition frequency is too far off from this value, the spherical resonance (plasma ball formation) cannot be reached. The exact value of the frequency fluctuates logically because the phase speed of sound depends on the state of the propagation medium. Assuming the bulb is an isothermal solid sphere and assuming the dissociation is at thermodynamic equilibrium, this parameter was calculated as a function of temperature in order to find the series of temperatures at which resonance would occur [2]. The mean temperature inside the actual bulb was assessed from the only doublet of this series that has characteristic frequencies close enough to cause the observed beats. In addition, one of these two modes has a spherical symmetry that can explain the plasma ball formation.

The experimental setup is similar to the one depicted in [2], as shown in Fig. 3. The plasma brightness is monitored by a photosensor whose bandwidth was set to $10 \mathrm{MHz}$. The microwave frequency is $2.45 \mathrm{GHz}$, which is non-tunable, and we only consider the pulsed steady-state regime, where the energy balance is fulfilled for the bulb as well as for the plasma ball, as the energy out- and in-flows counter balance each other over a pulsing period.

John P. Koulakis et al. have reproduced the plasma ball formation with a similar setup, but with a spinning bulb in 

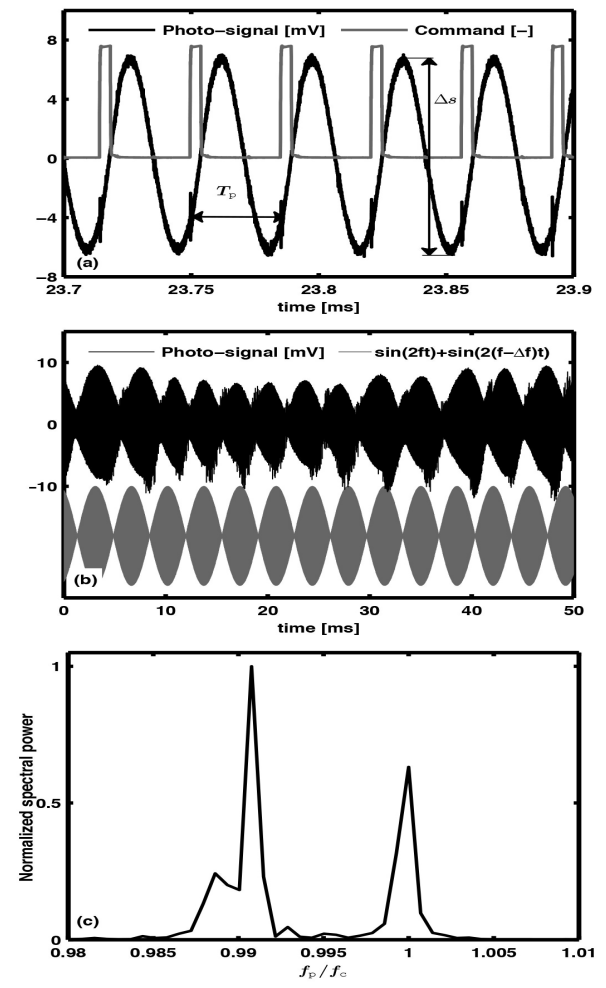

Fig. 2. (a) AC component of the photo signal (black) and the command signal (gray) of a plasma in spherical resonance. (b) Photo-signal of a spherical resonance of the plasma (black) and the sum of two sinusoids, where one has the frequency of $28.2 \mathrm{kHz}$ and the other has $1 \%$ lower frequency (gray). (c) Normalized spectral power of the photo signal. The horizontal axis is the frequency normalized with the repetition frequency $f_{c}$.

order to stabilize the plasma ball [6]. In agreement with our findings [2], they have also observed that the modulation of the light emission changes from sawtooth to sinusoidal shapes near an acoustic resonance. By high-speed videos, they have, moreover, been able to detect a sinusoidal variation of the plasma ball radius at the resonance frequency. They have observed that the light emission and the ball radius show almost exactly opposed phases. At resonance, the phase lag between the acoustic speed and pressure is expected to be close to $\pi / 2$ [7]. Hence, the molecular vibrational excitation due to the bulk viscosity, which is proportional to the square of the divergence of the speed, does not impact the light emission in a fast process in respect to the acoustic period. Besides, the degree of dissociation in the plasma results from the equilibrium between the reactions of dissociation and of recombination. So, a question arises: is the observed outphasing between the light emission and the ball radius related to shifts in chemical equilibrium that the acoustic oscillations may induce? On one hand, the Le Chatelier's principle says that a rise of pressure intensifies the reaction of recombination, because this direction of reaction tends to reduce the pressure. Then, the light flux would diminish soon afterward because of the resulting shift in equilibrium. On the other hand, the acoustic compression induces heating and the same principle says that this intensifies the dissociation, because the reaction is endothermic in this direction. The temperature, therefore, acts inversely to the pressure.

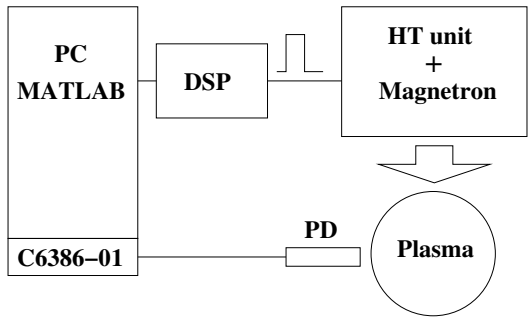

Fig. 3. Schematic of the experimental setup. PD: Photo diode; DSP: Digital Signal Processor; HT: High-voltage; PC: Personal computer.

As a general rule, the kinetics of chemical reactions is much slower than the vibrational relaxation [8]. In the previous publication [2], we have shown that the vibrational relaxation has a characteristic time of the same magnitude as the acoustic period, in the operating conditions. We also showed with power interruption tests that the decay time constant of the plasma luminous intensity, which reveals the plasma chemical kinetics, is about $9 \mathrm{~ms}$, i.e. more than 250 times the acoustic period. So, it can be assumed that the characteristic chemical time is much greater than the acoustic period, discrediting the importance of the direct effect of pressure and temperature on the modulation of plasma brightness. By this new modeling, we propose an alternative interpretation of the modulation, which is based on the effect of the plasma ball pulsation, i.e. the variation of its volume. In a simple approach, considering the plasma radiant exitance as constant, the light flux might be considered proportional to the ball surface, which would lead to in-phase oscillations, so contradicting the observations mentioned above. The model presented in this publication resolves this apparent contradiction.

In addition, John P. Koulakis et al. have calculated from the oscillation amplitude of the radius of the plasma ball that the sound level inside the bulb can exceed $180 \mathrm{~dB}$ ( re $20 \mu \mathrm{Pa}$ ). The improved model of the plasma ball presented in this publication allows one to calculate the acoustic amplitude solely from the measurement of the light modulation. A comparison with the result of John P. Koulakis et al. is furthermore achieved.

\section{A model to CAlCUlate the ACOUSTIC PRESSURE AMPLITUDE}

\section{A. Acoustic confinement}

In textbooks, one can find, e.g. in [9], the normal modes of oscillation in the case of a small amplitude in a homogeneous isothermal perfectly rigid solid sphere. The acoustic pressure in the fundamental spherical mode of resonance is given by

$$
p_{a}=P_{a} \frac{\sin (x)}{x} \cos (\omega t),
$$

where $t$ is the time, $\omega$ the angular frequency, $P_{a}$ the oscillation amplitude, $x$ the non dimensional radial coordinate equal to $\omega r / v=\chi r / a, v$ being the phase velocity of sound, $r$ the radial coordinate, $a$ the bulb radius $(1.55 \mathrm{~cm})$ and $\chi$ a constant due to the boundary conditions; $\chi=4.49341 \ldots$ assuming a rigid bulb [2]. 


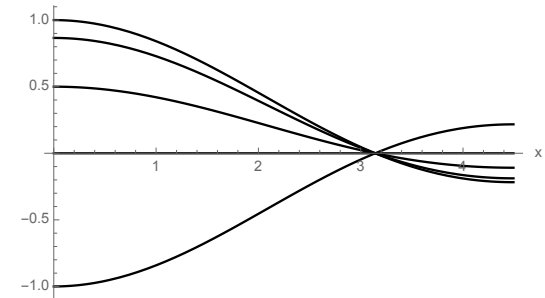

Fig. 4. Radial profile of the dimensionless acoustic pressure $p_{a} / P_{a}$ in the fundamental spherical mode (1), for $\omega t=\pi / 2, \pi / 6, \pi / 3, \pi / 2$ and $\pi$.

As the mean frequency of collisions of the free electrons with the molecules is an increasing function of the pressure, the absorption of microwaves also increases with the pressure, as it is observed during the warm-up phase. Therefore, the pressure profile in the fundamental spherical mode matches well with the acoustic confinement, leading to the formation of the plasma ball (cf. Fig. 4), as long as the pulsed microwaves are synchronized to the pressure rise at the center. Therefore, the acoustic pressure amplitude might depend on the frequency of the plasma oscillation. No phase lock was used in the experiments where the spherical mode has been observed, so the pulse frequency was constant for each sampling.

\section{B. Basis of the model}

First by the authors, a one-node-lumped model of the bulb was developed and reported in the preceding publication [2], where the temperature dependence of the velocity of sound is calculated to find the gas temperature and the average pressure inside the bulb. In the present publication this model is improved by accounting for the plasma ball formation to assess the acoustic pressure amplitude on the spherical resonance. Not only the geometry of the plasma is taken into account, but also the time variation of its volume. As shown in Fig. 1, the plasma ball is centered and extends to about half of the bulb radius. So we consider the plasma ball as an extendable sphere of about half size of the bulb (cf. Fig. 5).

As outlined in our first model, the bulb, in operating condition, is assumed to be filled with a chemically reacting mixture of ideal gases, because the degree of ionization is weak [1], page 14. The volume of the bulb as well as the enclosed mass, $m_{S}=28 \mathrm{mg}$ of sulfur and approximately $1 \mathrm{mg}$ of argon, are given constants. Unlike in the first model, we now consider the plasma separately from the rest of the bulb, and we take into account the variations of its volume (cf. Fig. 5). In the operational conditions the main chemical reaction is the dissociation and the recombination of disulfur $\mathrm{S}_{2} \leftrightarrow 2 \mathrm{~S}$ [1], page 14. Like in our first model [2], this is the only chemical equilibrium we account for, being aware of its predominance in the light emission mechanism [5], [10]. As seen before, the chemical characteristic time is higher than the acoustic period, so a luminous mass must belong to the plasma throughout such a time interval. Furthermore, given the slowness of molecular diffusion with respect to the sound propagation, we consider the plasma as a closed system on

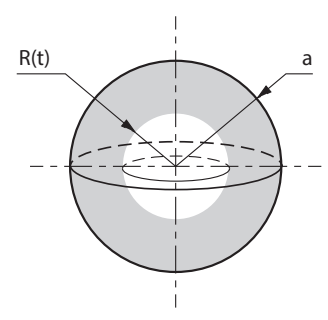

Fig. 5. Schematics of the plasma ball enclosed in the bulb; $a=15.5 \mathrm{~mm}$ $; \overline{R(t)} \approx a / 2$. The ball is distinguished from the rest of the interior by its luminescence.

the time scale of the acoustic process. We assume it to be composed of three chemical species whose numbers are:

1) $N_{A}$ atoms of argon $\mathrm{Ar}$,

2) $N_{1}$ atoms of sulfur $\mathrm{S}_{1}$,

3) $N_{2}$ diatoms of sulfur $\mathrm{S}_{2}$.

Note that the sulfur cannot exist in the crystalline form in these conditions [1] because of the high temperature of almost $2000^{\circ} \mathrm{C}$, see Table I.

Furthermore, if there was no dissociation, the number of sulfur diatoms would be constant and equal to $N_{0}=N_{2}+$ $N_{1} / 2$. The proportion between the two sulfur chemical species is given by the degree of dissociation $\alpha$

$$
N_{2}=N_{0}(1-\alpha) \text { and } N_{1}=2 N_{0} \alpha .
$$

Hence, the total number of molecules of sulfur is

$$
N_{2}+N_{1}=N_{0}(1+\alpha) \text {. }
$$

Furthermore, the plasma extends from the center to a volume with a radius of about half of the bulb size. The volumetric average of the pressure in the plasma ball of volume $V$, denoted by $p$, is decomposed into the acoustic pressure, whose amplitude is denoted by $\tilde{P}$, and a DC level $\bar{p}$, which might vary only slowly comparing to $\cos (\omega t)$ :

$$
p=\bar{p}+\tilde{P} \cos (\omega t) .
$$

By integrating (1) over the domain of the plasma ball, we obtain

$$
\tilde{P}=\frac{4 \pi P_{a} a^{3}}{\chi^{3} V} \int_{0}^{\chi R(t) / a} x \sin (x) d x .
$$

Noting that the amplitude of the ball pulsation is small in comparison to the ball size, we make the following approximation.

$$
\begin{aligned}
\tilde{P} & \approx \frac{4 \pi P_{a} a^{3}}{\chi^{3}(4 / 3) \pi(a / 2)^{3}} \int_{0}^{\chi / 2} x \sin (x) d x \\
& =\frac{24 P_{a}}{\chi^{3}}(\sin (\chi / 2)-(\chi / 2) \cos (\chi / 2)) .
\end{aligned}
$$

Hence, the ratio of the ball pressure amplitude $\tilde{P}$ to the acoustic pressure amplitude $P_{a}$ is

$$
\frac{\tilde{P}}{P_{a}} \approx \frac{24}{\chi^{3}}(\sin (\chi / 2)-(\chi / 2) \cos (\chi / 2))=0.578 \ldots
$$




\section{Acoustic perturbations}

The plasma extends over a domain with volume $V$ throughout which the average of temperature, denoted by $T^{1}$, is assumed to be related to the degree of dissociation, also supposed to be uniform, according to the ideal gas law:

$$
\frac{p}{T}=\frac{N_{A}+N_{0}(1+\alpha)}{V} .
$$

In this model, the plasma ball has a variance of 3 because it is assumed to be a homogeneous closed system of 3 components, constrained by one stoechiometric relation $d N_{1}=-2 d N_{2}$ [11]; i.e., the plasma only depends on three independent variables that we choose to be $V, \alpha$, and $N_{A}$; hence, its pressure fluctuations are such that

$$
d p=\left.\frac{\partial p}{\partial V}\right|_{\alpha, N_{A}} d V+\left.\frac{\partial p}{\partial \alpha}\right|_{V, N_{A}} d \alpha+\left.\frac{\partial p}{\partial N_{A}}\right|_{V, \alpha} d N_{A} .
$$

However, because the plasma is considered in the present model as a closed system over a period of acoustic oscillation, $N_{A}$ is constant

$$
d p=\left.\frac{\partial p}{\partial V}\right|_{\alpha} d V+\left.\frac{\partial p}{\partial \alpha}\right|_{V} d \alpha
$$

As acoustic fluctuations usually are faster than heat transfer processes, we can assume that the compression - expansion cycles are adiabatic, in agreement with the high values of quality factor found experimentally as mentioned in [2]. It follows, as derived in the Appendix, that

$$
\left.\frac{\partial p}{\partial V}\right|_{\alpha}=-\gamma \frac{p}{V}
$$

where $\gamma$ is the isentropic coefficient, which is defined as the ratio of the isobaric to the isochoric heat capacities, $c_{p}$ and $c_{v}: \gamma=c_{p} / c_{v}$.

Moreover, assuming that the content of the ball is a mixture of ideal gases, we decompose the pressure into the partial pressures of each component: argon, $p_{A}=N_{A} T / V$, monoatomic sulfur, $p_{1}=2 N_{0} \alpha T / V$, and diatomic sulfur, $p_{2}=N_{0}(1-\alpha) T / V$, such that the total pressure is

$$
p=p_{A}+p_{0}(1+\alpha)
$$

where $p_{0}$ is the sulfur partial pressure if there was no dissociation. It is determined by

$$
p_{0}=N_{0} \frac{T}{V} .
$$

The other partial derivative in (10) is now obtained by (12)

$$
\left.\frac{\partial p}{\partial \alpha}\right|_{V}=p_{0}
$$

and, finally, (10) yields the relative differential of the plasma ball pressure

$$
\frac{d p}{p}=\frac{p_{0}}{p} d \alpha-\gamma \frac{d V}{V} .
$$

${ }^{1}$ We simplify the equations by omitting to multiply the temperature by the Boltzmann constant.
TABLE I

DC CHARACTERISTICS AT THE MEAN ACOUSTIC TEMPERATURE [2]

\begin{tabular}{lccc}
\hline name & symbol & value & unit \\
\hline \hline mean acoustic temperature & $T_{B}$ & 1930 & ${ }^{\circ} \mathrm{C}$ \\
pressure & $p_{B}$ & 523 & $\mathrm{kPa}$ \\
equil. constant of dissociation & $K_{e}$ & 16.6 & $\mathrm{~Pa}$ \\
degree of dissociation in LTE & $\alpha_{e q}$ & 0.28 & $\%$ \\
frozen isentropic coefficient & $\gamma_{\infty}$ & 1.28 & \\
equil. isentropic coefficient & $\gamma_{0}$ & 1.19 & \\
molar mass & $\bar{M}$ & 63.6 & $\mathrm{~g} / \mathrm{mol}$ \\
sulf. density if there were no disso. & $n_{0 B}$ & $1.6910^{19}$ & $\mathrm{~cm}^{-3}$ \\
\hline
\end{tabular}

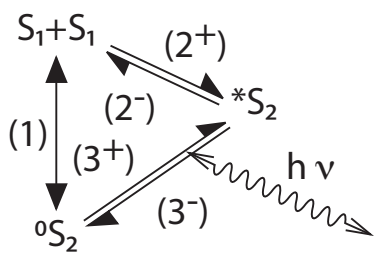

Fig. 6. Model of photoemission by chemical cycling: each electronic relaxation from the excited state $B^{3} \sum_{u}^{-}$(labeled ${ }^{*} S_{2}$ ) to the ground state $X^{3} \sum_{g}^{-}$(labeled ${ }^{o} S_{2}$ ) generates a photon in the optical spectral range $(h \nu)$.

\section{Kinetics}

In the operating system, the pressure in the bulb rises slightly above 5 bars (cf. Table I). Then the weakly ionized sulfur vapor absorbs efficiently the $2.45 \mathrm{GHz}$ microwaves, and the dissociation of sulfur becomes relevant [2]. The emission of light is mainly due to the chemical cycling of dissociation and recombination of sulfur dimer; the recombination produces dimers in an electronic excited state of radiative deexcitation: $\mathrm{S}_{1}+\mathrm{S}_{1} \rightarrow{ }^{*} \mathrm{~S}_{2} \rightarrow{ }^{\mathrm{o}} \mathrm{S}_{2}+\mathrm{h} \nu$ [1], page 15, [5], [10].

In order to determine the dynamics of the excited dimer state ${ }^{*} S_{2}$ (cf. Fig. 6), we now consider the kinetics of each of the reactions involving this state, i.e. reactions $\# 2^{+}, \# 2^{-}$, $\# 3^{+}$and $\# 3^{-}$, discussing independently each of them. The dissociation results from electronic impacts as well as from pure vibrational mechanisms, but in the spherical acoustic resonance it is driven by the second process, as we have shown by power interruption tests [2]. Focusing on the population of excited dimers, we have therefore

$$
N+{ }^{*} S_{2} \rightarrow N+S_{1}+S_{1},
$$

where $N$ is a neutral. This reaction gives the following rate equation

$$
\frac{d^{*} N_{2}}{d t}=-k_{2}^{-*} n_{2} n_{N}
$$

where $k_{2}^{-}$is the reaction rate coefficient of the reaction $\# 2^{-}$ (cf. Fig. 6), ${ }^{*} n_{2}={ }^{*} N_{2} / V$ the number density of the excited dimer, and $n_{N}$ the number density of the neutral buffers given as

$$
n_{N}=\frac{N_{N}}{V}=\frac{N_{A}+N_{1}+N_{2}}{V} .
$$


The recombination process occurs mostly at the location of the collision between two atoms of sulfur [12]

$$
S_{1}+S_{1} \rightarrow{ }^{*} S_{2}
$$

The net rate of dissociation-recombination of the excited dimer is hence

$$
\frac{d^{*} N_{2}}{d t}=k_{2}^{+} n_{1}^{2}-k_{2}^{-*} n_{2} n_{N}
$$

where $k_{2}^{+}$is the reaction rate coefficient of the reaction $\# 2^{+}$ (cf. Fig. 6). The radiative electronic transitions are of zerothorder for the spontaneous emission and second-order for the absorption

$$
{ }^{*} S_{2} \rightleftharpoons{ }^{o} S_{2}+h \nu,
$$

as well as for the stimulated emission

$$
h \nu+{ }^{*} S_{2} \rightarrow{ }^{o} S_{2}+2 h \nu .
$$

In contrast to higher-order reactions, a zeroth-order reaction is one whose rate is independent of the concentration of the reactant. The plasma ball being considered as a closed system, the oscillation of its volume induces a variation of concentrations. So, to be in agreement with the measurements of John P. Koulakis et al., [6], the light modulation is likely to be governed by the stimulated emission. Hence, taking into account also the absorption, we have

$$
\frac{d^{*} N_{2}}{d t}=\left(k_{3}^{+}{ }^{0} n_{2}-k_{3}^{-*} n_{2}\right) n_{p},
$$

where $n_{p}$ is the number density of the photons, $k_{3}^{+}$and $k_{3}^{-}$ are the reaction rate coefficients of the absorption and the stimulated emission (cf. Fig. 6).

Now, let's balance the excited dimer population from the flow due to radiative transfers (23) and the flow due to dissociation-recombination (20)

$$
\frac{d^{*} N_{2}}{d t}=k_{2}^{+} n_{1}^{2}-k_{2}^{-*} n_{2} n_{N}+\left(k_{3}^{+o} n_{2}-k_{3}^{-*} n_{2}\right) n_{p} .
$$

Considering moreover the plasma as quasi optically thick (see page 3 in [2]), we actually cancel the two reactions involving $n_{p}$ in the balance equation

$$
\frac{d^{*} N_{2}}{d t}=k_{2}^{+} n_{1}^{2}-k_{2}^{-*} n_{2} n_{N}
$$

According to [1], page 20, the plasma of the sulfur lamp with a turning bulb is optically thick between 200 and $400 \mathrm{~nm}$ and almost fully transparent above $550 \mathrm{~nm}$. However, measured spectra were compared with calculations, and a number of differences were found which may be due to an incorrectly modelled temperature profile or non-local thermal equilibrium (LTE) effects [10]. With an improved model, notably taking into account the presence of $S_{3}$, this research group has become able to quantitatively describe the position of the spectral maximum and to predict its shift due to the pressure variation [13]. In our case, nevertheless, the bulb is static and the microwave flow is pulsed, unlike in the former case, so that the plasma is in acoustic spherical resonance. As a result, the temperature profile could be somewhat different and the deviation from LTE also. Therefore, our thick plasma hypothesis does not contradict previous results.
By measuring the decay rate of the fluorescence at 370 $\mathrm{nm}$, McGee et al. have determined the lifetime of the excited dimer state $\left(B^{3} \sum_{u}^{-}\right)$to be $\tau_{B}=45 \mathrm{~ns}$ [14], whereas in [15] a value $20 \mathrm{~ns}$ was communicated. Because this is far below the acoustic period $2 \pi / \omega$, we may assume that the population of the excited state, ${ }^{*} S_{2}$, is quasi stationary. Applying to (25), we obtain

$$
k_{2}^{-} n_{N}^{*} n_{2} \approx k_{2}^{+} n_{1}^{2}
$$

Admitting the reaction rate coefficients as constants, we are lead to

$$
n_{N}{ }^{*} n_{2} \propto n_{1}^{2},
$$

which yields

$$
{ }^{*} n_{2} \propto n_{1}^{2} / n_{N}=n_{1} N_{1} / N_{N} .
$$

With (18) and (2) and taking into account that the Argon is a minor additive, $N_{A} \ll N_{0}$, the inverse ratio on the right-hand side reads

$$
N_{N} / N_{1} \approx \frac{1+\alpha}{2 \alpha} .
$$

Applying to (28) we obtain

$$
{ }^{*} n_{2} \propto \frac{2 \alpha}{1+\alpha} \frac{N_{1}}{V} .
$$

Because the emission spectrum is stable as well as the sensitivity of the photodiode that monitors the brightness of the plasma ball, in a good approximation, we can admit that the signal of the photodiode, $y$, is proportional to the flux of photons. Hence, since the stimulated emission is supposed to govern the light modulation, (22) yields

$$
y \propto k_{3}^{-} n_{p}{ }^{*} n_{2} .
$$

Since the photons are not dragged along by the molecules, the concentration of a given number of photons is independent of the ball volume $V$. In addition, knowing that the modulation of the signal is weak compared to its average value, the characteristic time of the reaction $\# 3^{-}$is supposed to be much longer than the acoustic period and, on this account, the rate of production of photons is supposed to be constant during this time scale. Consequently by this two arguments, the number density $n_{p}$ is assumed to be constant, and so

$$
y \propto{ }^{*} n_{2} .
$$

Furthermore, applying (30) and (2) and noting that $N_{0}$ is constant leads to

$$
y \propto \frac{\alpha^{2}}{1+\alpha} \frac{1}{V} .
$$

Therefore, the relative differential of the signal is

$$
\frac{d y}{y} \propto \frac{2+\alpha}{1+\alpha} \frac{d \alpha}{\alpha}-\frac{d V}{V} .
$$

Since $0 \leq \alpha \leq 1$, we note that $3 / 2<(2+\alpha) /(1+\alpha)<2$. Thus, this factor does not change the order of magnitude of the effect of dissociation on the relative differential of the signal $d y / y$. Hence, assuming in addition $\alpha$ sufficiently constant so that

$$
\left|\frac{d \alpha}{\alpha}\right| \ll\left|\frac{d V}{V}\right|,
$$




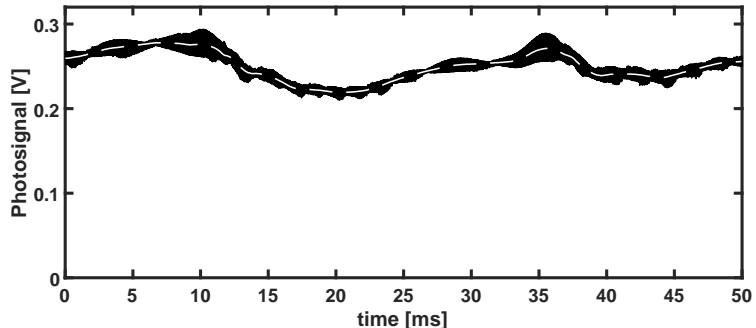

Fig. 7. A sampling of the plasma brightness variation in time: view of the whole record. The trace thickness is due to the modulation of the light emission. The DC level is shown with the white dashed line.

we obtain according to (34)

$$
\frac{d y}{y} \approx-\frac{d V}{V} .
$$

Since $0 \geq \alpha \geq 1$, we have

$$
d \alpha \leq \frac{d \alpha}{\alpha} .
$$

Hence, (35) provides us with

$$
|d \alpha| \ll\left|\frac{d V}{V}\right| .
$$

Besides $p>p_{0}$ and $\gamma>1$, so that

$$
1<\frac{\gamma p}{p_{0}} .
$$

In multiplying the two last inequalities, we obtain

$$
d \alpha \ll \gamma \frac{p}{p_{0}}\left|\frac{d V}{V}\right|,
$$

which according to (15), yields

$$
\frac{d p}{p} \approx-\gamma \frac{d V}{V} .
$$

This result shows that, in fact, the light emission intensity oscillates on opposed-phase with respect to its radius, thus in agreement with the measurements of John P. Koulakis et al. [6]. After substitution of Eq. (36) into (41), this yields

$$
\frac{d p}{p} \approx \gamma \frac{d y}{y} .
$$

\section{Application to experimental data}

\section{A. Signal post processing}

This section presents the numerical process applied to the signal delivered by the photodiode in order to calculate the term $d y / y$ that appears in (42). An example of such a digital signal is shown in Fig. 7 with a zoom, showing a node, presented in Fig. 8.

The post processing decomposes the signal in two components:

- the DC level, i.e., the mean value during one cycle,

- the AC component, i.e. the signal minus the DC level.

As shown in Fig. 2(a), the frequency of the plasma response is $f_{p}=1 / T_{p}$, with $T_{p}$ being the period of the light intensity oscillation. However, a difficulty arises due to a slight aperiodicity in the brightness modulation. Even though the

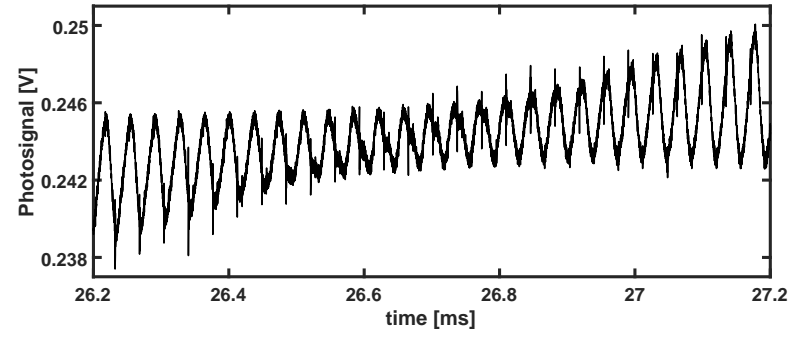

Fig. 8. A sampling of the plasma brightness variation in time: zoom on a node.

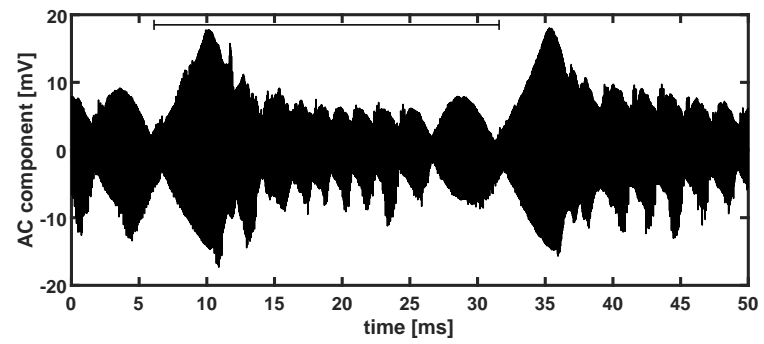

Fig. 9. AC component of the plasma brightness versus time; the envelope shows the amplitude modulation. The horizontal line indicates one super period.

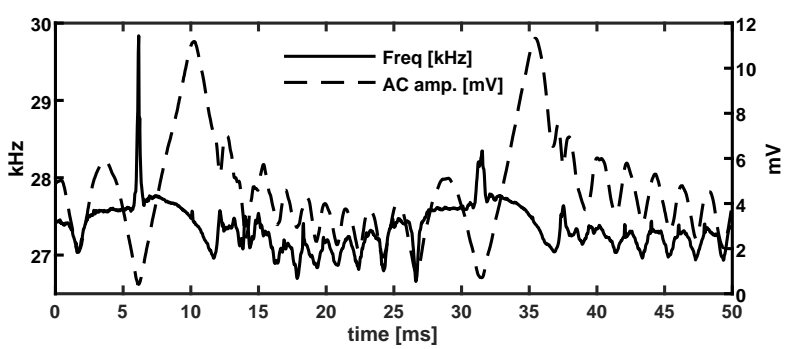

Fig. 10. Frequency and amplitude of the AC component of the plasma brightness vs. time. The microwave pulse repetition frequency is $27.7 \mathrm{kHz}$.

light oscillation of the plasma in resonance is very close to a sinusoid at the time scale of a few microwave pulses, on the longer term, one can observe that, in contrast to the pulses, it is actually not strictly periodic. So, in a first step, the DC level is calculated in using the periodicity of the pulses instead of $T_{p}$. Then, the obtained AC component (cf. Fig. 9) is taken to determine the actual periodicity of the response $T_{p}$ by its zero crossings. This operation requires an interpolation of the samples in order to avoid aliasing. The use of linear interpolation at zero-crossings leads to satisfactory results.

Figs. 9 and 10 show the decomposition of the sampling of Fig. 7. It clearly appears that the beats in this sampling are not as regular as in the sampling shown in Fig. 2, which was used in our first analysis [2]. Here, the signal shows a super periodicity comparable to the one found by John P. Koulakis et al. (cf. Fig. 3 of [6]). In both experiments this super frequency lies in the same range, between 30 and $70 \mathrm{~Hz}$. In our experiments, this super frequency range corresponds to $400-850$ acoustic periods while a figure of 500 acoustic periods is reported in [16]. It seems, therefore, that this feature 


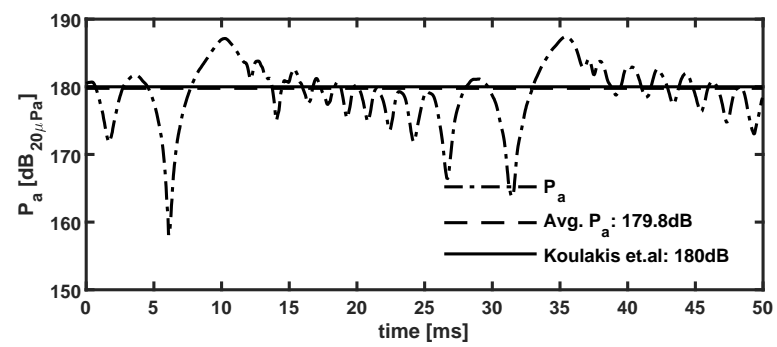

Fig. 11. Acoustic pressure versus time according to our two-nodes-lumped new model and comparison of the average value over a super-period $(25,3$ $\mathrm{ms}$ ) with the level found by John P. Koulakis et al. (180 dB re $20 \mu \mathrm{Pa}$ ).

reveals a characteristic time, which has been interpreted in [16] as a relaxation oscillation emanating from sudden rises of the thermal transport to the bulb caused by periodic plasma eruptions. Finally, as one can see in Fig. 7, the modulation amplitude stays small in respect to the DC level. Hence, to a good approximation, we assume that $d y / y$ is equal to the ratio of the efficient (R.M.S.) value of the $\mathrm{AC}$ component to the DC level.

In a preceding study, the authors have assessed the characteristic parameters given in Table I. They are the averages over the entire inner domain of the bulb, when the plasma ball formation is occurring [2]. On this basis, we now calculate the acoustic amplitude in the bulb in the spherical resonance regime.

\section{B. Acoustic amplitude}

In this section we use the sampling shown in Fig. 7 to determine the acoustic amplitude by (42). The value of the isentropic coefficient is taken from Table I, and it is according to [2] assumed that the acoustic wave periodically put the molecular vibrational excitation of the plasma back and forth between the equilibrium state and the frozen state. $\gamma \approx\left(\gamma_{\infty}+\right.$ $\left.\gamma_{0}\right) / 2=1.24$.

By Eq. (42), admitting by extrapolation that $d p / p=\tilde{P} / \bar{p}$, and furthermore applying (7)

$$
P_{a}=\frac{\gamma \bar{p}}{0.578} \frac{d y}{y} .
$$

A mechanical equilibrium tends to uniformize static pressure. The chronometric average (DC level) of pressure, $\bar{p}$, is taken from Table I - a value that has been calculated in [2] from the measurement of the frequency of resonance. In putting the results of (43) on the decibel scale, as done in Fig. 11, we observe that the result (discontinuous line) fluctuates and shows a periodic pattern that reaches the value $180 \mathrm{~dB}$ (re $20 \mu \mathrm{Pa}$ ) given by John P. Koulakis et al. [6].

The acoustic pressure has been calculated for a series of plasmas using (43). With the DC pressure level of $523 \mathrm{kPa}$ for all measurements and taking the time average of $P_{a}$ over one super period, we found the average values of acoustic pressure shown in Fig. 12. The length of the super period is indicated below the symbols in [ms]. Five cases (pls 2, pls 3, pls 4, pls 5, and pls 9) have a super periodicity visible both in the DC level and AC component of the plasma brightness,

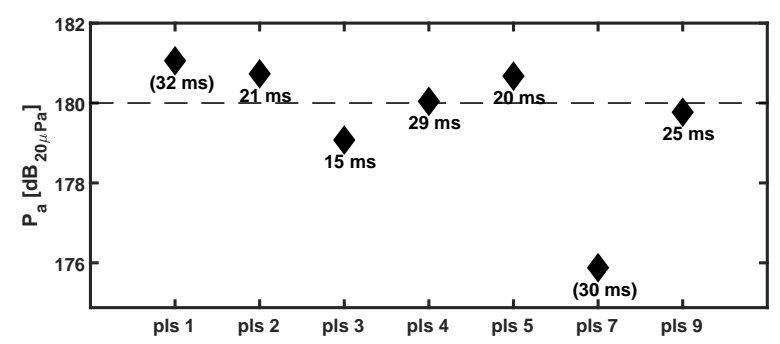

Fig. 12. Average pressure over a super period for seven plasmas calculated with the new model from the photosensor signal. The numbers below the symbols show the length of the super period in [ms]. The dashed line is at $180 \mathrm{~dB}$.

as shown in Figs. 7 and 9. For pls 1 and pls 7 the super periodicity manifests itself only in the DC level while the AC signal only shows beating of two modes, as shown in Fig. 2(b). Therefore, for these two plasmas, the value is in parenthesis. The super periodicity varies between $15 \mathrm{~ms}$ and $32 \mathrm{~ms}$, and it is remarkable that pls 1 and pls 7 are also distinguished by the two longest super periodicities. In averaging the acoustic pressure on a super period, we obtain values between 179.1 $\mathrm{dB}$ and $181.1 \mathrm{~dB}$ except for pls 7 which has a lower pressure amplitude of $176 \mathrm{~dB}$, but a super periodicity appearing only in the DC level. We conclude that our result meets the one that John P. Koulakis et al. have obtained from displacement measurements of the plasma ball boundary with an ultra-fast camera [6].

\section{CONCLUSiON}

In our previous publication [2] we have studied the phenomenon of a plasma ball formation in the on-going development of a $1 \mathrm{~kW}$ microwave sulfur plasma lamp with a static bulb. In that work we concluded that the plasma ball formation is due to central absorption of the microwave pulses. The plasma confinement was accounted for a spontaneous excitation of a spherically symmetric standing acoustic wave with a pressure anti-node (maximum of the acoustic pressure) at the spherical bulb center, therefore, we call this phenomenon the spherical resonance. We were able to assess for the temporal and volumetric average of the plasma pressure a value of $523 \mathrm{kPa}$. One of the limitations of this previous model is that the acoustic medium in the bulb was supposed homogeneous, as we had adopted a one-node-lumped model.

The present work is an extension to this previous description of the plasma in the spherical resonance. While the plasma geometry is assumed to be spherical, we can now take into account the time variations of the plasma radius, i.e., the pulsations of the plasma volume, Fig. 5, as well as the pressure variations, Eq. (15), due to the acoustic oscillations within the adiabatic approximation. In addition, the chemical reaction kinetics are taken into account. The main chemical reactions are recombination of mono-atomic sulfur and dissociation of the diatomic sulfur molecule. The light modulation is mainly attributed to the stimulated photon emission of the excited diatomic sulfur molecule.

Considering the reaction rates as constants and modeling the plasma as an optically thick closed system where the chemical 
kinetics are sufficiently s low $\mathrm{w}$ ith $\mathrm{r}$ espect $\mathrm{t}$ o $\mathrm{t}$ he acoustic oscillations of the plasma for the inequality (35) to be fulfilled, we obtained Eqs. (36) and (42). This is one of the main results of the publication. These equations state that the volume of the plasma ball and its brightness oscillate with the acoustic frequency but with a phase difference of $\pi$, a result which is in agreement with the measurements by John P. Koulakis et al. [6] observed in a similar plasma. This qualitative agreement with the measurements is a first indication that our description of the plasma ball in spherical resonance is a realistic one.

Applying the relation between the plasma brightness and the pressure (42) to the fast plasma brightness measurements, by using the average pressure found in [2], results in values of the acoustic amplitudes in good agreement with the level reported by John. P. Koulakis et al. [6], as shown in Fig. 11. The model presented in this paper, therefore, states that the phase opposition of the plasma volume and the brightness oscillations could be due to the stimulated emission. It is mentioned in [6] that measuring the oscillations of the luminous radius gives only a lower bound of the acoustic pressure, because compression/expansion cycling of gas near the light emission threshold partially offsets the acoustic motion. With a chemically frozen gas on the acoustic time scale, inequality (35), our model therefore also gives the lower limit of the average sound level. Thus, the quantitative agreement between the results should not be taken as a validation of this kinetic hypothesis, nor as an indication that the sound level we found is in fact not exceeded. It would be interesting to complete this work with measurements to test the assumptions made in the modeling, especially regarding the optical thickness.

\section{APPENDIX}

\section{DEMONSTRATION OF EQUATION 11}

The heat exchange for an infinitesimal transformation of an ideal gas is [17]

$$
\delta Q_{I G}=c_{v} V d p+c_{p} p d V
$$

However, our plasma model is an ideal reacting gas mixture; thus we consider in addition the energy of dissociation, whose molar value is noted by $D$

$$
\delta Q=c_{v} V d p+c_{p} p d V-N_{0} D d \alpha
$$

which for an adiabatic transformation at constant dissociation leads to

$$
c_{v} V d p=-c_{p} p d V
$$

Using Eq. (10), we obtain

$$
\left.c_{v} V \frac{\partial p}{\partial V}\right|_{\alpha} d V=-c_{p} p d V
$$

which finally gives

$$
\left.\frac{\partial p}{\partial V}\right|_{\alpha}=-\frac{c_{p}}{c_{v}} \frac{p}{V}
$$

\section{ACKNOWLEDGMENT}

This work was supported by the Swiss National Science Foundation with funding (contract CRSK-2_190619). The authors would like to thank also the company Lumartix, who has provided specific components of the prototype.

The measured data is available at https://zenodo.org/record/4288800\#.X70mcsIo-Cg

\section{REFERENCES}

[1] C. W. Johnston, "Transport and equilibrium in molecular plasmas: the sulfur lamp," Ph.D. dissertation, Technische Universiteit Eindhoven, 2003.

[2] G. Courret, P. Nikkola, S. Wasterlain, O. Gudozhnik, M. Girardin, J. Braun, S. Gavin, M. Croci, and P. W. Egolf, "On the plasma confinement by acoustic resonance," Eur. Phys. J. D, vol. 71, no. 214, 2017.

[3] L. Horath, Fundamentals of Materials Science for Technologists, 3rd ed. IL, USA: Waveland Press, 2009.

[4] M. T. Sebastian, H. Jantunen, and R. Ubic, Microwave Materials and Applications, 1st ed. UK: John Wiley \& Sons, 2017.

[5] H. W. P. van der Heijden and J. J. A. M. van der Mullen, "Semiclassical and quantum-mechanical descriptions of s2 molecular radiation," $J$. Phys. B: At. Mol. Opt. Phys., vol. 34, pp. 4183 - 4201, 2001.

[6] J. P. Koulakis, S. Pree, A. L. F. Thomton, and S. J. Putterman, "Trapping of plasma enabled by pycnoclinic acoustic force," Phys. Rev. E, vol. 98, no. 043103, 2018.

[7] P. M. Morse and K. U. Ingard, Theoretical Acoustics, 1st ed. Princeton, N.J., USA: Princeton University Press, 1968.

[8] A. I. Osipov and A. V. Uvarov, "Kinetic and gasdynamic processes in nonequilibrium molecular physics," Sov. Phys. Usp., vol. 35, p. 904, 1992.

[9] L. E. Kinsler, A. R. Frey, A. B. Coppens, and J. V. Sanders, Fundamentals of acoustics, 4th ed. N.J., USA: John Wiley \& Sons, 2000.

[10] H. van der Heijden, J. van der Mullen, J. Baier, and A. Körber, "Radiative transfer of a molecular $\mathrm{s} 2 \mathrm{~b}-\mathrm{x}$ spectrum using semiclassical and quantum-mechanical radiation coefficients," J. Phys. B: At. Mol. Opt. Phys., vol. 35, p. 3633 - 3654, 2002.

[11] A. Kastler, Thermodynamique. France: Editions Masson \& Cie , 1962, page 444 .

[12] J. Meichsner, M. Schmidt, R. Schneider, and H.-E. Wagner, Nonthermal Plasma Chemistry and Physics, 1st ed. FL, USA: CRC Press, 2013, pages $74-75$.

[13] C. W. Johnston, H. W. P. van der Heijden, A. Hartgers, K. Garloff, J. van Dijk, and J. J. A. M. van der Mullen, "An improved lte model of a high pressure sulfur discharge," J. Phys. D, vol. 37, pp. 211 - 220, 2004.

[14] T. H. McGee and R. E. Weston, "Lifetime of the b3 sigma u state of s2," Chem. Phys. Lett., vol. 47, pp. 352 - 356, 1977.

[15] A. Fridman and L. A. Kennedy, Plasma Physics and Engineering, 2nd ed. CRC Press USA, 2011, page 77.

[16] S. Pree, J. P. Koulakis, A. L. F. Thomton, and S. J. Putterman, Acoustoconvective relaxation oscillation in plasma lamp. Santa $\mathrm{Fe}$, New Mexico, USA: 21st International Symposium on Nonlinear Acoustics, 2018, vol. 34

[17] R. Brun and N. Belouaggadia, Thermodynamique, physique statistique et cinétique. Paris, France: Ellipses, 2018, page 32.

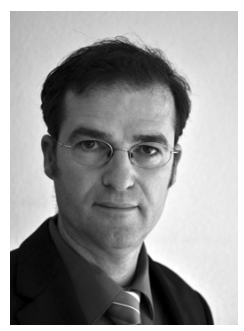

Gilles Courret obtained the Engineering degree in physics and material sciences of the National Institute of Applied Sciences (INSA) of Lyon, France, in 1988. He received the Ph. D. degree of the Swiss Federal Institute of Technology (EPFL) in 1999.

Since 2013, he is Professor of physics in the Department of Industrial Technologies of the University of Applied Sciences and Arts Western Switzerland (HES-SO). His research interests include microwave-plasma interaction, plasma chemistry, light sources and illumination engineering, with emphasis on the improvement of energy efficiency. 


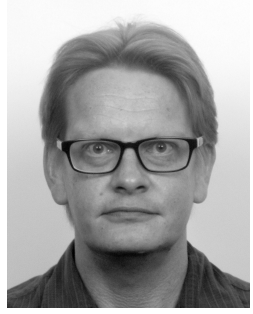

Petri Nikkola was born in Helsinki Finland in 1971. He got his M.Sc. (Tech.) degree in 1998 in the Helsinki University of Technology (currently Aalto Yliopisto). In 2004 he received the Ph.D. degree from the Swiss Federal Institute of Technology (EPFL), in the field of nuclear fusion research. He is a collaborator at the Institute of Energy and Electrical Systems at the Department of Industrial Technologies of the University of Applied Sciences and Arts Western Switzerland (HES-SO). Currently $\mathrm{He}$ is working in various applied research projects.

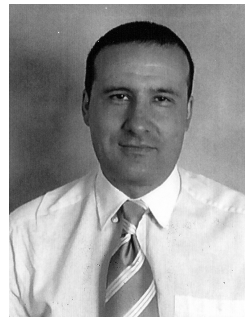

Mirko Croci was born in Geneva, Switzerland, in 1960. In 1980 he obtained the HES-Master in electrical engineering of the Engineering School of Geneva, the M.S. degree in Physics of the University of Geneva in 1985 and the Ph.D. degree in 1992 from the Swiss Federal Institute of Technology (EPFL). Since 2002, he is Professor of physics in the Department of Industrial Technologies of the University of Applied Sciences and Arts Western Switzerland (HES-SO). From 2004 to 2014, he founded and led the Laboratory of Applied Physics for Emerging Technologies. His research interests include plasma physics, nanotechnologies and renewable enery sources. He is now actively working in the "Institut de Génie Thermique" led by Prof. Nicolas Weber.

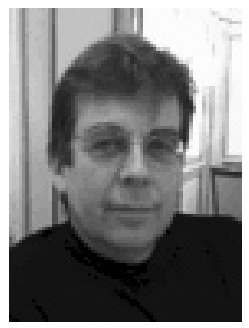

Peter W. Egolf received the Heating, Air Conditioning, and Refrigeration Engineer's Diploma degree from the Lucerne University of Applied Sciences and Arts, Horw, Switzerland, in 1977, the Physics Diploma degree in dynamical meteorology in 1984 and the Ph.D. degree in low-temperature physics in 1990 from the Swiss Federal Institute of Technology Zurich (ETHZ), Zurich, Switzerland. Until 1999, he was a Researcher with the Swiss Federal Institute for Materials Testing and Research (EMPA) developing and investigating sustainable and energy-saving

systems.

From 2000 to 2018, he was Professor and Head of the Theory and Numerical Simulations Division (SIT) of the Thermal Sciences and Engineering Institute of the University of Applied Sciences and Arts of Western Switzerland, Yverdon-les-Bains, Switzerland.

Since 2019, he is at the Institute of Theoretical Turbulence Research in Niederlenz, Switzerland, studying nonlinear, nonlocal and fractional turbulence. For more information on his biography, see Who's Who in the World 2020. 